Z. Phys. Chem. 216 (2002) 555-574

(C) by Oldenbourg Wissenschaftsverlag, München

\title{
Fluorescence Excitation Spectrum, Lifetimes and Photoisomerization of Jet-Cooled Conformers of 1,1'-bi(benzocyclobutylidene)
}

By D. Ernst ${ }^{1}$, L. Rupp ${ }^{1}$, R. Frank ${ }^{2}$, W. Kühnle ${ }^{3}$, P. Lemmen ${ }^{4}$, D. Lenoir ${ }^{2}$, J. Schroeder ${ }^{1,3, *}$, Ch. Grimm ${ }^{1}$ and T. Steinel ${ }^{1}$

${ }^{1}$ Institut für Physikalische Chemie der Universität, Tammannstr. 6, D-37077 Göttingen, Germany

${ }^{2}$ Institut für Ökologische Chemie, GSF-Forschungszentrum Neuherberg, D-85758 Oberschleißheim, Germany

${ }^{3}$ Max-Planck-Institut für Biophysikalische Chemie, Am Fassberg, D-37077 Göttingen, Germany

${ }^{4}$ Institut für Organische Chemie und Biochemie, Technische Universität München, Lichtenbergstr. 4, D-85747 Garching, Germany

Dedicated to Prof. Dr. Dr. h.c. mult. Jürgen Troe on the occasion of his 60th birthday

(Received August 20, 2000; revised version accepted March 30, 2001)

\section{Fluorescence Excitation Spectra / 1,1'-bi(benzocyclobutylidene) / Photoisomerization}

First measurements of fluorescence excitation spectra of the recently synthesized rigid stilbene analogue 1,1'-bi(benzocyclobutylidene) in a supersonic jet expansion show that, in contrast to the parent compound, both the trans- and the cis-conformer fluoresce under these conditions. The excitation energy dependence of fluorescence lifetimes indicates the onset of an efficient non-radiative decay channel above energy thresholds of $1340 \mathrm{~cm}^{-1}$ and $990 \mathrm{~cm}^{-1}$ for the trans- and cis-form, respectively, which is assigned to photoisomerization in the singlet state. From an RRKM analysis of the microcanonical rate coefficients an estimate of the high pressure limit of the thermal photoisomerization rate coefficient is obtained and compared with photoisomerization rate coefficients measured in low viscosity solution and in thermal vapor. There are strong indications that for this compound there are no dynamic or static solvent induced effects that lead to an anomalous acceleration of the reaction in solution.

* Corresponding author. E-mail: jschroe2@gwdg.de 


\section{Introduction}

The isomerization of stilbene (1) and related compounds upon excitation to the $S_{1}$ state has served as a prototype trans-cis-photoisomerization reaction to study fundamental aspects of gas and condensed phase kinetics and dynamics for several decades [1-12]. Among the major issues in the photochemical kinetics of trans-1 there are two puzzling observations that have occupied experimentalists and theoreticians alike. (1) An unexpectedly weak dependence of the photoisomerization rate coefficient $k$ in liquid solution on solvent viscosity $\eta$ of the type $k \sim \eta^{-\alpha}$ with $\alpha \leq 0.5$ was found by varying viscosity either in homologous solvent series (1a) $[13,14]$ or in compressed single solvents (1b) [15]. (2) An order of magnitude discrepancy appeared between rate coefficients $k$ measured in low viscosity supercritical solution in the vicinity of the so-called Kramers turnover region [16-18] and the calculated high pressure limit of the rate coefficient $k_{\infty}[19-21]$ based on experimentally determined specific rate constants $k(E)$ of isolated trans-1 [22-26]. As the interpretation of these phenomena in terms of the underlying microscopic dynamics or slight changes of the effective potential energy surface is still discussed controversially, it is important to know whether they are related to specific structural properties of trans-1 or rather the consequence of general physical effects playing an important role in many photochemical processes. For this purpose comparative studies of chemically suitably modified compounds covering an equally wide range of experimental conditions from the isolated molecule and low pressure gas phase to supercritical and compressed liquid solution are required to assess the relevance of the seemingly anomalous behavior found for trans-1. The only similar molecule for which such extensive studies of photoisomerization rate coefficients exist is 1,4-diphenyl-(1,3)-butadiene (2).

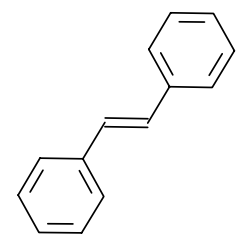

1

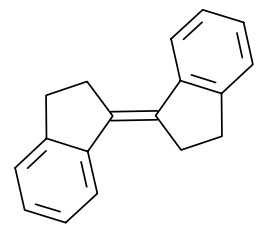

3

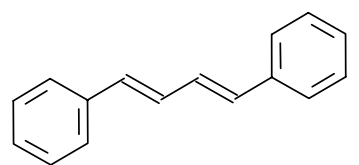

2

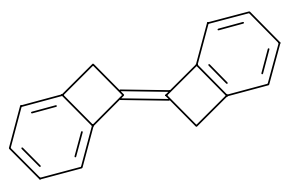

4

While measurements of rate constants in homologous solvent series resulted in a $k(\eta)$ similar to that for trans-1 [27], measurements of the pressure de- 
pendence in various liquid solvents [28, 29] resulted in $k \sim \eta^{-1}$, as expected from the Smoluchowski limit of Kramers theory [30,31]. Furthermore, the high pressure limit $k_{\infty}$ as calculated from $k(E)$ obtained from measurements in supersonic beam expansions of 2 [32-34] agrees to within a factor of less than two with rate coefficients $k$ of photoisomerization in low viscosity fluids [35]. These findings seem to indicate that the weak viscosity dependence of $k$ in $n$-alkane and $n$-alkanol solvents series (1a) represent a more general phenomenon suggesting that microviscosity effects involving the relative size of solute and solvent molecules [36,37] may be responsible for the deviation of $k(\eta)$ from $\eta^{-1}$-behavior under these conditions [38-41]. The absence of phenomena (1b) and (2) in the photoisomerization of $\mathbf{2}$, however, is an indication that they might be closely connected and that particulars of the potential energy surface of trans $\mathbf{- 1}$ could be responsible for its abnormal behavior.

It would be very desirable to test this view by looking more closely at other related molecules for which the $k\left(\eta^{-1}\right)$ dependence of photoisomerization rates in compressed solvents was determined, like cis-1 [42], tetraphenylethylene [43], or 1-(1-indanylidene)indane (3) (so-called "stiff" stilbene) [44]. Unfortunately, in all these cases the very low fluorescence quantum yield under isolated molecule conditions prevented fluorescence lifetime measurements in the supersonic jet $[45,46]$. Recently, however, Schneider et al. reported measurements of the pressure and temperature dependence of fluorescence lifetimes in $n$-hexane and methylcyclohexane for the novel bridged stilbene derivative bi(benzocyclobutylidene) (4) [47]. They found a weak $k(\eta)$-dependence similar to that observed of trans-1, but their analysis of the different isotherms of $k(\eta)$ led them to conclude that the isoviscous activation energy $E_{\mathrm{A}}$ of photoisomerization of trans $\mathbf{- 4}$ was practically independent of viscosity, having values of 12.8 and $12.5 \mathrm{~kJ} / \mathrm{mol}$ in $n$-hexane and methylcyclohexane, respectively. For trans-1, in contrast, it was found that $E_{\mathrm{A}}$ varies with solvent viscosity in compressed $n$-hexane [15] and $n$-pentane [48], which was taken as evidence for solvent induced lowering of the height of the reaction barrier with increasing density of the compressed liquid solvent. As it was suggested that a corresponding effect on the barrier height could be among the causes of the order of magnitude discrepancy between $k_{\infty}$ and the reaction rates observed in low viscosity fluid solution $[17,49]$, it would be extremely interesting to measure $k(E)$ of $\mathbf{4}$ in a supersonic jet expansion and obtain the barrier height for the isolated molecule. One could then calculate $k_{\infty}$ by using a suitable harmonic model of $\mathbf{4}$, and compare its value with rate constants measured in the gas phase at pressures ranging from about 1 to 100 bar.

The bridged stilbene 4 at the same time offers the possibility to investigate the aspect of dimensionality of the barrier crossing process [50-59]. As it was suggested that the anomalous viscosity effect in the case of trans-1 could be caused by the effective multidimensionality of motion involving the phenyl torsion coordinate [60] and quantum chemical calculations revealed a significant variation of the effective barrier to photoisomerization in the $S_{1}$-state [61], 
stilbene derivatives in which the phenyl rings are fixed by linking them covalently to the ethylenic carbon atoms are of particular interest. One might suspect that reducing the dimensionality of motion along the reaction path by blocking phenyl torsion could lead to qualitative changes in the dynamics of the reaction.

In this paper we present laser induced fluorescence excitation spectra of jetcooled conformers of $\mathbf{4}$ and the excess energy dependence of their fluorescence lifetimes. We present an RRKM-analysis of measured $k(E)$ based on preliminary harmonic models to determine the barrier height $E_{0}$ for the reaction and estimate the high pressure limit of the photoisomerization rate coefficient $k_{\infty}$.

\section{Experimental}

\subsection{Supersonic beam expansion}

As most details of the experimental set-up have been described elsewhere [62], we only summarize the main features including some new details where required. The jet apparatus consists of a vacuum chamber evacuated by a diffusion pump (Edwards Diffstak 250/2000 M) backed by a rotary pump (Edwards E2M40). The samples were heated to $90-150^{\circ} \mathrm{C}$, entrained in $1.5-10$ bar helium and expanded into the vacuum using a General Valve Series 9 pulsed nozzle. The nozzle orifice had a diameter of $100 \mu \mathrm{m}$.

\subsection{Fluorescence measurements}

\section{Excitation Spectra}

For measuring fluorescence excitation spectra, the jet beam is crossed at right angles by a perpendicularly polarized laser beam. The laser system consists of a dye-laser (Lambda Physik Scanmate II, $0.15 \mathrm{~cm}^{-1}$ resolution) pumped by a Nd:YAG laser (Quantel Brilliant $\omega$ ) at a $10 \mathrm{~Hz}$ repetition rate. The dyelaser beam was frequency doubled in a KDP crystal to generate excitation wavelengths in the range from 268 to $325 \mathrm{~nm}$. The excitation laser pulses (duration $6 \mathrm{~ns}$, bandwidth $0.2 \mathrm{~cm}^{-1}$, pulse energy about $20 \mu \mathrm{J}$ ) were focused onto the beam expansion, using a lens of $500 \mathrm{~mm}$ focal length, at about $10 \mathrm{~mm}$ downstream from the nozzle, corresponding to a reduced distance of 100. At stagnation pressures of 5-10 bar and nozzle open times of $1 \mathrm{~ms}$, the background pressure in the chamber could be kept close to $5 \mathrm{mPa}$. The broadband fluorescence was collected perpendicularly to the plane spanned by laser beam and jet expansion and focused by a collimating $f / 1$ lens system onto a microchannel plate photomultiplier (MCP-PMT, Hamamatsu R3809U). The resulting signal was accumulated in a custom built boxcar integrator (integration time typically $100 \mu \mathrm{s}$ ) and digitized using a WIN30D-A/D-PCAT card. Typically the signal was averaged over 10 to 50 laser shots. Normalization 
with respect to laser intensity was used only when pulse to pulse fluctuations as measured by a photodiode exceeded a factor of two. Prior to measuring fluorescence decays, excitation spectra were taken at a reduced spectral resolution of about $4 \mathrm{~cm}^{-1}$ using the picosecond laser system and a continuous jet expansion (see below).

\section{Lifetimes}

Fluorescence lifetimes were measured using a time correlated single photon counting apparatus described in detail previously $[28,62,63]$. The excitation source was a synchronously pumped (Coherent Antares 76S Nd:YLF pump laser) dye laser (home built) tunable by a three-plate birefringent filter (Coherent) giving pulses of $10-15 \mathrm{ps}$ duration and a few $\mathrm{nJ}$ energy at a bandwidth of about $4 \mathrm{~cm}^{-1}$. These were frequency doubled in a $\mathrm{LiJO}_{3}$ or $\mathrm{BBO}$ crystal to obtain tunable excitation pulses in the wavelength range $285-315 \mathrm{~nm}$. Wavelength calibration was achieved by optogalvanic spectroscopy using a He atom lamp.

The excitation pulses were focused onto the continuous jet expansion about $5 \mathrm{~mm}$ downstream from the nozzle, corresponding to a reduced distance of 67 . For these experiments, the stagnation pressure was close to $3 \mathrm{bar}$, resulting in a background pressure in the vacuum chamber of less than $20 \mathrm{mPa}$.

Fluorescence was collected as described above by the MCP, whose output pulses were amplified (Hamamatsu C5594 preamplifier, $36 \mathrm{~dB}, 1.5 \mathrm{GHz}$ ), fed into a constant fraction discriminator (Tennelec TC454) and used as start pulses for the time-to-amplitude converter (Tennelec TC862) operated in reverse mode. Stop pulses were obtained from an avalanche photodiode monitoring the excitation laser. The TAC output was digitized by a fast Wilkinson A/D-converter (Nuclear Data ND582) at 12 bit resolution and accumulated in a PC via a digital input PC-AT-card (Meilhaus ATDIO-32-F).

The instrument response function was determined as $45 \pm 10$ ps by laser light scattering off air dust particles in the aerated vacuum chamber. Decay curves were analyzed by a least-squares convolution and fitting routine using the Levenberg-Marquardt algorithm.

\subsection{Synthesis}

\section{Preparation of 1,1'-bi(benzocyclobutylidene) (4)}

Previously, on a small scale trans $\mathbf{- 4}$ and $c i s-\mathbf{4}$ were prepared by Wittig-reaction and manual sorting of the crystals [64] or by McMurry-reaction and separation by preparative HPLC [65]. They were also obtained, among other compounds, by photochemical decomposition of benzocylobutanone tosyl hydrazone [66].

For the current investigation a larger quantity of pure trans-4 was needed. We found, that crystallization of the trans-/cis-mixture, obtained by McMurry reaction $[67,68]$, from a saturated solution of picric acid in ethanol afforded the pure picric acid charge transfer complexes of trans $\mathbf{- 4}$ and of enriched cis-4. 
Trans- and cis-1,1'-bi(benzocyclobutylidene) (trans-4 and cis-4)

A mixture of trans- and cis-4, obtained by McMurry reaction and flash chromatography [47] (0.92 g, trans-/cis-ratio $=63 / 37)$ was crystallized repeatedly from a saturated ethanolic solution of picric acid. Crystal crops were analyzed for their trans-/cis-ratio by NMR-spectroscopy and combined accordingly to yield $2.21 \mathrm{~g}$ orange crystals (trans-/cis-ratio $=99 / 1$, ratio picric acid $/$ trans $-\mathbf{4}$ $=2.6$ ) and $1.6 \mathrm{~g}$ red needles (trans-/cis-ratio $=10 / 90)$. Both crystal crops were distributed between dichloromethane and saturated sodium bicarbonate solution (4 steps, countercurrent), the organic phase dried $\left(\mathrm{Na}_{2} \mathrm{SO}_{4}\right)$, evaporated and the residue recrystallized from ethanol to yield $0.40 \mathrm{~g}$ pure trans -4 (m.p. $181-182^{\circ} \mathrm{C}$ ) (Lit.: $178-181{ }^{\circ} \mathrm{C}$, [64]) and $0.33 \mathrm{~g}$ of a mixture enriched in cis-4 (trans- $/$ cis-ratio $=12 / 88)$ (m.p. $\left.120-124^{\circ} \mathrm{C}\right)\left(\right.$ Lit.: $124-126^{\circ} \mathrm{C}$, [64]).

\section{Results}

\subsection{LIF excitation spectra}

Based on the observation that only the trans-conformer of $\mathbf{1}$ fluoresces under isolated molecule conditions [69] and only trans-4 was reported to show significant fluorescence in liquid solution [47], we started from the assumption that this also holds for isolated conformers of $\mathbf{4}$. We expected that in an expansion of a cis-4 enriched sample containing about 10 percent trans-4 we would observe only the LIF excitation spectrum of trans-4. We took several LIF excitation spectra in a row from such an expansion while the pulsed gas flow was maintained. The sample temperature was constant at $80^{\circ} \mathrm{C}$, leading to a gradual enrichment of trans $\mathbf{- 4}$ in the beam because the melting point of cis-4 is about $60{ }^{\circ} \mathrm{C}$ lower than that of trans-4. The spectra are normalized to the peak intensity of the first transition at $E_{\mathrm{e}}=0$, corresponding to the energy of the 0-0-transition of trans $\mathbf{- 4}$ (see below). If only trans-4 was fluorescent, all the spectra should be equal, which is clearly not the case. In contrast, a significant decrease of the initially most intense line in Fig. 1 reflected the gradual decrease of $\mathrm{cis} \mathbf{- 4}$ in the expansion and indicated that this conformer fluoresces significantly after excitation at wavelengths quite distinct from those of trans-4. Separate high resolution spectra of pure trans-4 and enriched cis-4 in the low excess energy region clearly support this conclusion, as illustrated in Fig. 2, and show that the 0-0-transitions of both conformers are separated by $(91.1 \pm 0.6) \mathrm{cm}^{-1}$. Their absolute positions are $(32147.6 \pm 0.3) \mathrm{cm}^{-1}$ and (32237.7 \pm 0.3$) \mathrm{cm}^{-1}$ for trans-4 and cis $\mathbf{- 4}$, respectively.

The trans-4 excitation spectrum could be measured with high resolution at low excess energy over the range indicated in Fig. 3. A low resolution spectrum covering excess energies up to $2500 \mathrm{~cm}^{-1}$ is shown in Fig. 4. The excitation spectrum of enriched $c i s-\mathbf{4}$ was obtained with low resolution only up to an excess energy of about $800 \mathrm{~cm}^{-1}$. Higher energy transitions of the cis-conformer 


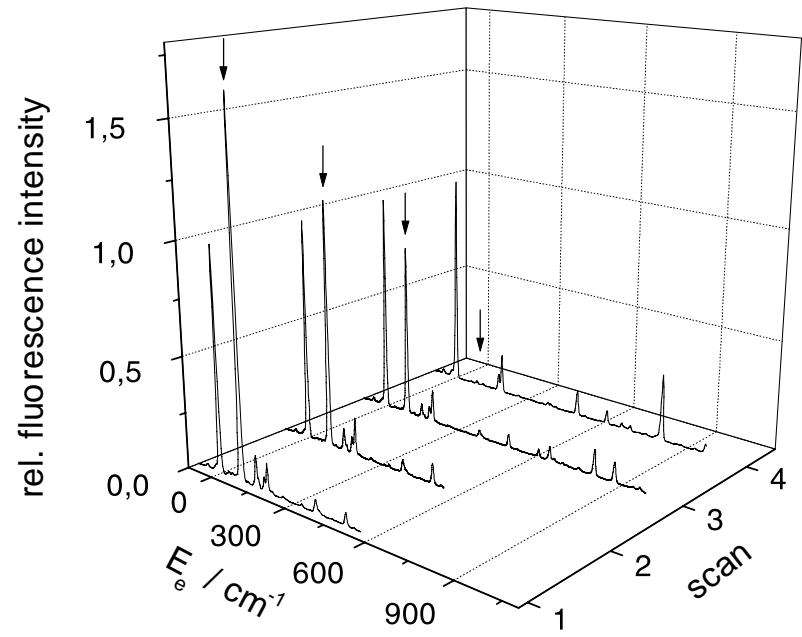

Fig. 1. Successive scans of the LIF excitation spectrum of jet-cooled 4.

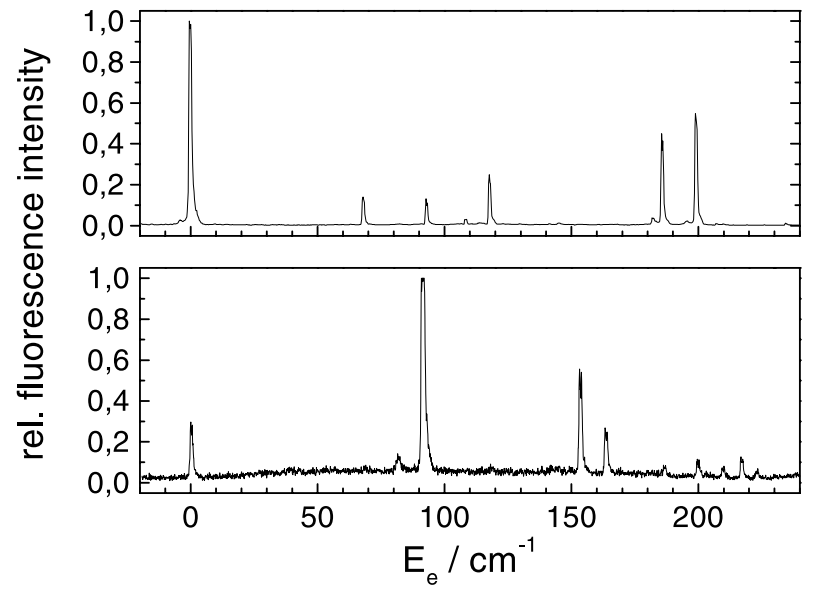

Fig. 2. Low frequency part of the LIF excitation spectra of jet-cooled "pure" trans-4 (top) and "enriched" cis-4. Energy scale with respect to the 0-0-transition of trans-4.

were identified by comparing LIF excitation spectra from expansions of the mixture with those of pure trans-4. Thus, Fig. 5 shows an excitation spectrum of cis-4 which at excess energies higher than $850 \mathrm{~cm}^{-1}$ represents a difference spectrum between the mixture and pure trans-4. In Figs. 4 and 5, line intensities are normalized to those of the respective 0-0-transition. In the cis-4-spectrum, some artifacts resulting from subtraction of the trans-4-spectrum appear that are marked by asterisks. 


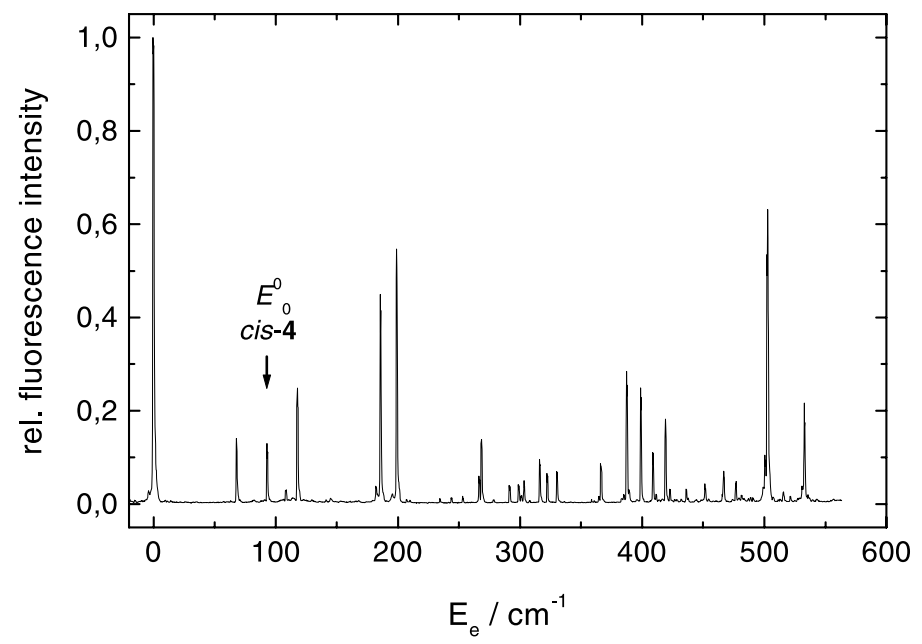

Fig. 3. High resolution $\left(0.2 \mathrm{~cm}^{-1}\right)$ LIF excitation spectrum of jet-cooled trans-4.

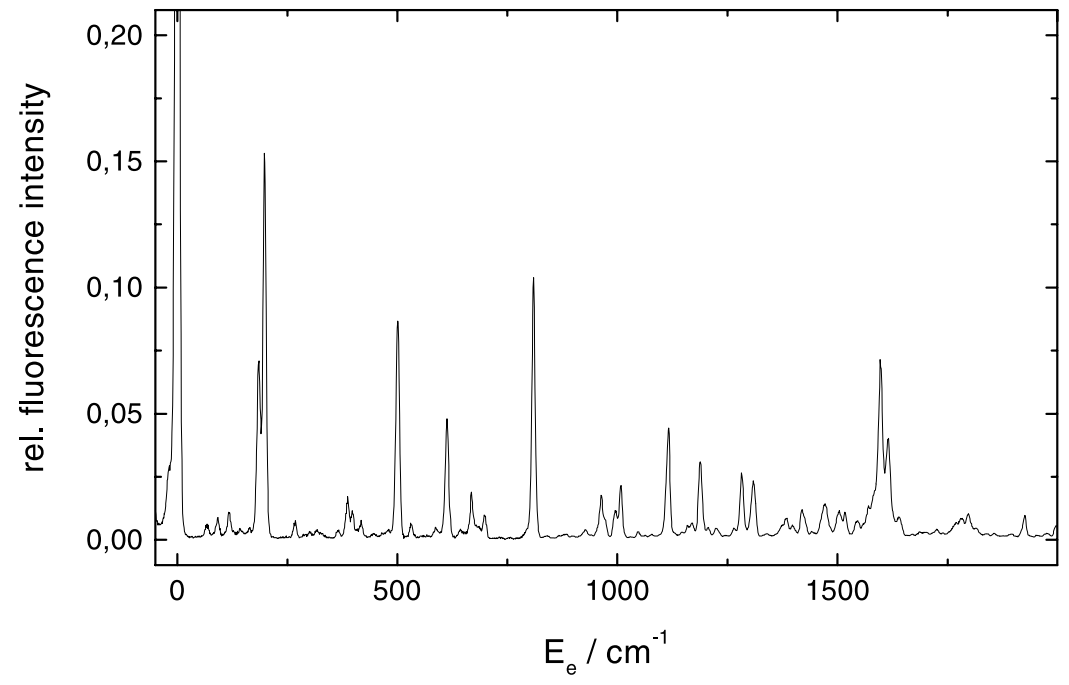

Fig. 4. Low resolution $\left(4 \mathrm{~cm}^{-1}\right)$ LIF excitation spectrum of jet-cooled trans-4.

\subsection{Fluorescence lifetimes of jet-cooled trans- and cis-4}

Lifetimes were measured in expansions of pure trans $\mathbf{- 4}$ and of the mixture of conformers. In the region of low excess energy the assignment of observed lines to transitions of either cis-4 or trans-4 was verified by comparing the corresponding fluorescence lifetimes: in the low excess energy region, the $S_{1}$ 


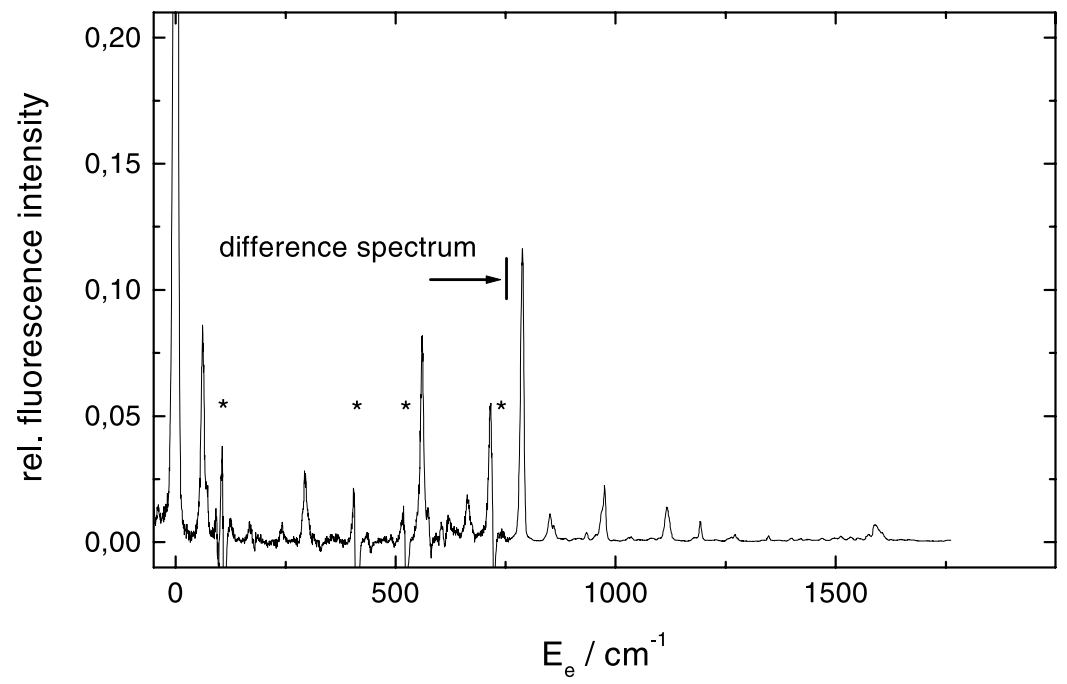

Fig. 5. Low resolution $\left(4 \mathrm{~cm}^{-1}\right)$ LIF excitation spectrum of jet-cooled cis-4. The low frequency part (up to $750 \mathrm{~cm}^{-1}$ ) represents a difference spectrum of the conformer mixture and trans $\mathbf{- 4}$, as indicated. Subtraction artefacts are indicated by stars.

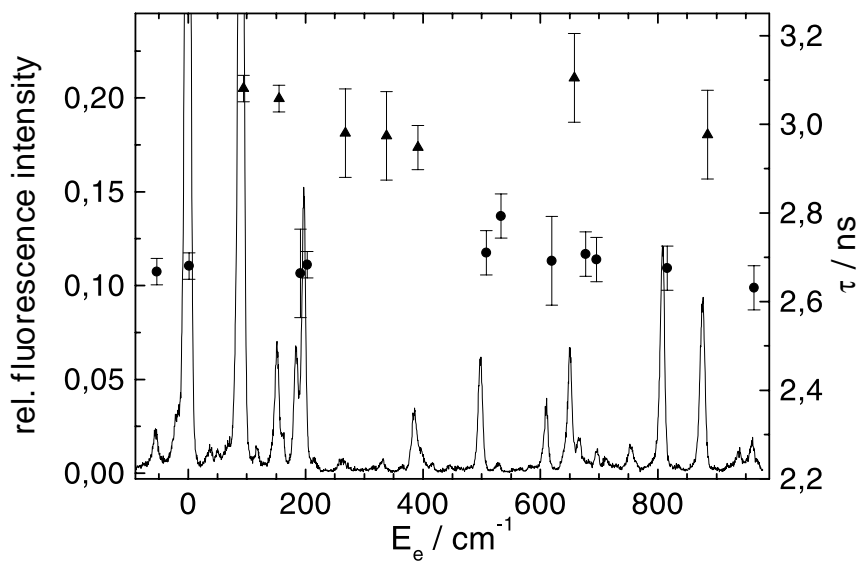

Fig. 6. Fluorescence lifetimes obtained from convoluting and fitting a mono-exponential decay function to measured single photon counting decay traces in a jet-cooled conformer mixture at low excess energies. The LIF excitation spectrum is shown for comparison.

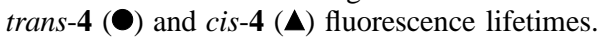

lifetimes of the trans-conformer varied between 2.6 and $2.7 \mathrm{~ns}$, while those of the cis-conformer were close to $3 \mathrm{~ns}$, as illustrated in Fig. 6 . The fluorescence lifetimes after exciting the $0-0$-transitions were found to be $2.68 \pm 0.05 \mathrm{~ns}$ for trans $\mathbf{- 4}$ and $3.08 \pm 0.05 \mathrm{~ns}$ for cis $\mathbf{- 4}$. 


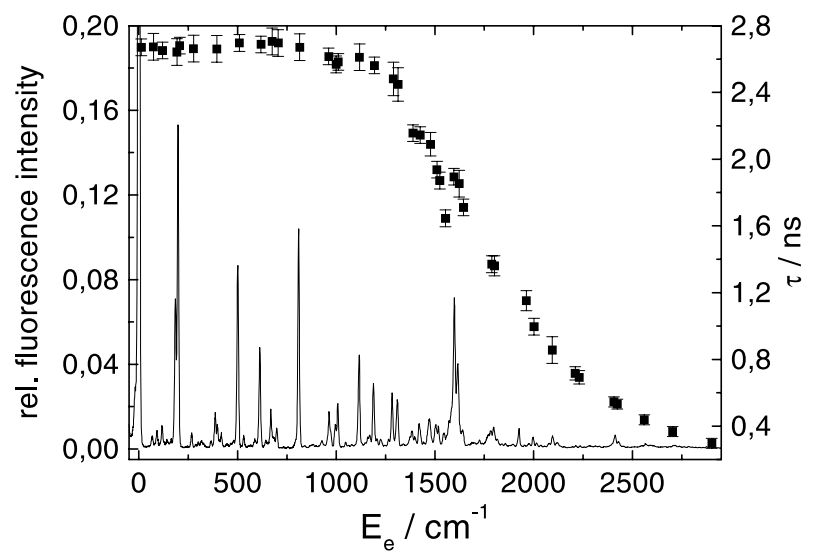

Fig. 7. Excess energy dependence of mean fluorescence lifetimes of jet-cooled trans-4

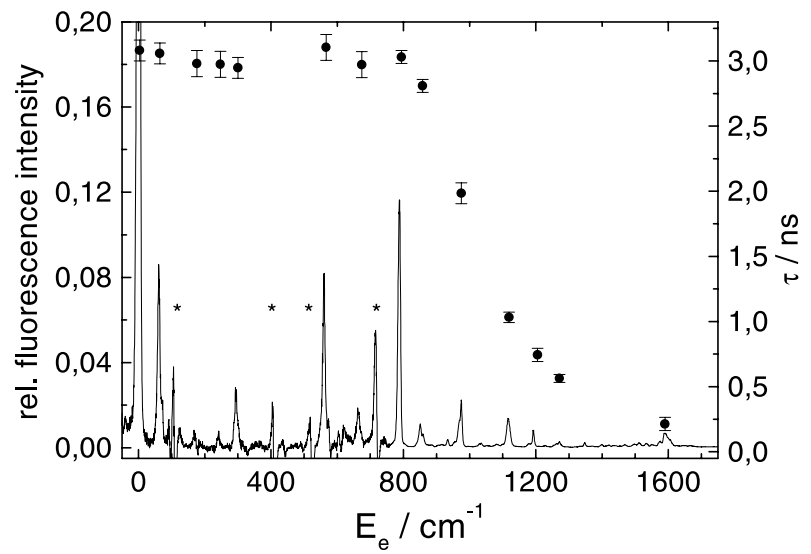

Fig. 8. Excess energy dependence of mean fluorescence lifetimes of jet-cooled cis-4

At low excess energy $E_{\mathrm{e}}=\tilde{v}_{\mathrm{ex}}-E_{0}^{0} \leq 1300 \mathrm{~cm}^{-1}$, trans $-\mathbf{4}$ fluorescence decays were strictly single exponential, whereas in the range $1300 \mathrm{~cm}^{-1}<E_{\mathrm{e}}<$ $2400 \mathrm{~cm}^{-1}$ they could only be fitted by a sum of two exponentials. The possible reasons for this behavior have been discussed in detail for the case of trans $\mathbf{- 1}$ [62], and most probably are related to the J-dependence of the reaction rate at energies close to the barrier. Consequently, in the critical energy range the values displayed in Fig. 7 are average fluorescence lifetimes defined as

$$
\bar{\tau}=\frac{A_{1} \tau_{1}+A_{2} \tau_{2}}{A_{1}+A_{2}}
$$


where $A_{i}$ is the amplitude of the $i$ th decay component and $\tau_{i}$ its lifetime. For the cis-conformer the decays start to be double exponential at excess energies $E_{\mathrm{e}}>800 \mathrm{~cm}^{-1}$, the corresponding fluorescence lifetimes are shown in Fig. 8.

\section{Discussion}

As the main point of interest in the present context is the excess energy dependence of fluorescence lifetimes, we will not discuss the LIF excitation spectrum of trans-4 in detail and discuss the possible assignment of the observed transitions. In Table 1, we list the main observed LIF excitation lines with at least 3 percent of the intensity of the $0-0$-transition in the high and about 0.5 percent in the low resolution spectrum. For comparison, we also include results published for matrix-isolated trans $\mathbf{- 4}$ in argon at $15 \mathrm{~K}$ [70]. In general, one may note that the overall intensity of transitions with respect to the $0-0$-transition is lower than for trans-1 [25, 26, 62], and there are no prominent progressions. This is not surprising, because of the significantly more rigid structure of trans-4 [70] which is planar in $S_{0}$ and $S_{1}$ singlet states. An interesting exception is the fairly strong transition at $810 \mathrm{~cm}^{-1}$ reappearing with pronounced overtones at around 1620 and $2430 \mathrm{~cm}^{-1}$. which is absent in trans-1 as well as styrene [71] and may be related to a low frequency mode of the cyclobutane/benzene subunit. In Table 1, we include a very tentative classification of lines as arising from fundamentals, combinations, and overtones. The main surprise, however, is that jet-cooled cis-4 shows fluorescence just slightly less intense in comparison to the trans-conformer. The quality of the LIF excitation spectrum, apart from the very low excess energy region, is rather poor, though, precluding a precise determination of line positions. There are fewer strong lines than for trans $\mathbf{- 4}$, but the prominent transition at $790 \mathrm{~cm}^{-1}$ is also present.

Both LIF excitation spectra rapidly decrease in intensity above an excess energy of about $1700 \mathrm{~cm}^{-1}$ for trans-4 and $1000 \mathrm{~cm}^{-1}$ for cis-4 which is accompanied by a corresponding decrease of fluorescence lifetimes. This indicates the onset of an efficient significantly excess energy dependent nonradiative decay process which - on the basis of available photochemical data [47] - we assign to singlet state photoisomerization in analogy to trans-1. The magnitude of the rate coefficient $k_{\mathrm{nr}}$ for this process is estimated from

$$
k_{\mathrm{nr}}=\frac{1}{\tau_{\mathrm{fl}}}-\frac{1}{\tau_{\mathrm{fl}, 0}}
$$

where $\tau_{\mathrm{fl}, 0}$ is the average fluorescence lifetime at low excess energy. For trans -4 the mean value in the excess energy range $0 \leq E_{\mathrm{e}} \leq 810 \mathrm{~cm}^{-1}$ is $(2.68 \pm 0.02) \mathrm{ns}$, while for cis-4 we find $(3.02 \pm 0.06) \mathrm{ns}$ in the interval $0 \leq E_{\mathrm{e}} \leq 790 \mathrm{~cm}^{-1}$. The rate constant $k_{0}=1 / \tau_{\mathrm{fl}, 0}$, which includes purely radiative decay as well as possibly minor contributions from internal conversion and 
Table 1. LIF excitation transitions and corresponding fluorescence lifetimes of trans-4.

\begin{tabular}{|c|c|c|c|c|c|c|}
\hline $\begin{array}{c}E_{\mathrm{e}} / \mathrm{cm}^{-1} \\
\text { (ns-LIF/jet) }\end{array}$ & $I_{\text {rel. }}$ & $\begin{array}{l}\text { Preliminary } \\
\text { classification }\end{array}$ & $\begin{array}{l}E_{\mathrm{e}} / \mathrm{cm}^{-1} \\
(\text { matrix })^{\mathrm{a})}\end{array}$ & $\begin{array}{c}E_{\mathrm{e}} / \mathrm{cm}^{-1} \\
\text { (ps-LIF/jet) }\end{array}$ & $I_{\text {rel. }}$ & $\tau_{\mathrm{ff}} / \mathrm{ns}$ \\
\hline 0 & 100 & $0_{0}^{0}$ & & 0 & 100 & $2.67(5)$ \\
\hline 68 & 14 & $f$ & & 68 & 0.6 & $2.67(8)$ \\
\hline 108 & 3 & $f$ & & & & \\
\hline 118 & 25 & $f$ & & 116 & 1 & $2.65(5)$ \\
\hline 186 & 45 & $f, 68+118$ & & 185 & 7 & $2.64(8)$ \\
\hline 199 & 55 & $f$ & 203 & 197 & 15 & $2.68(5)$ \\
\hline 266 & $6\}$ & $f$ & & 267 & 7 & $2.66(8)$ \\
\hline 268 & $14\}$ & $68+199$ & & & & \\
\hline 292 & 41 & $108+186$ & & & & \\
\hline 299 & $4\}$ & $f$ & & 300 & 3 & \\
\hline 303 & 5 & $118+186$ & & & & \\
\hline 316 & 10 & $118+199$ & & 317 & 0.4 & \\
\hline 322 & 7 & $f$ & & & & \\
\hline 330 & 7 & $f$ & & & & \\
\hline 366 & 9 & $68+297$ & & 366 & 0.4 & \\
\hline 387 & 28 & $186+199$ & & 387 & 2 & \\
\hline 389 & 3 & $68+322$ & & & & \\
\hline 399 & 25 & $2 \cdot 199$ & 406 & 397 & 1 & $2.66(8)$ \\
\hline 409 & 11 & f & & & & \\
\hline 419 & 18 & $f$ & & 417 & 0.7 & \\
\hline 423 & 3 & $2 \cdot 118+186$ & & & & \\
\hline 436 & 3 & $2 \cdot 118+199$ & & & & \\
\hline 451 & 4 & $186+267$ & & & & \\
\hline 467 & 7 & $199+267$ & & & & \\
\hline 477 & 5 & $68+409$ & & 480 & 0.4 & \\
\hline 501 & $11\}$ & $f$ & & & & \\
\hline 503 & $63\}$ & $118+186+199$ & 503 & 500 & 9 & $2.70(5)$ \\
\hline 516 & 3 & $186+330$ & & & & \\
\hline \multirow[t]{19}{*}{533} & 22 & $f$ & & 530 & 0.6 & \\
\hline & & $186+2 \cdot 199$ & & 586 & 0.5 & \\
\hline & & $f$ & 612 & 612 & 5 & $2.69(5)$ \\
\hline & & $f$ & 669 & 668 & 2 & $2.71(8)$ \\
\hline & & $199+501$ & 707 & 697 & 1 & $2.70(8)$ \\
\hline & & $f$ & 810 & 810 & 10 & $2.67(8)$ \\
\hline & & f & 970 & 963 & 2 & $2.62(5)$ \\
\hline & & $186+810$ & & 995 & 1 & $2.57(5)$ \\
\hline & & $199+810$ & & 1008 & 2 & $2.58(5)$ \\
\hline & & $118+2 \cdot 501$ & & 1117 & 4 & $2.61(8)$ \\
\hline & & $186+2 \cdot 501$ & & 1188 & 3 & $2.56(5)$ \\
\hline & & $f$ & & 1282 & 3 & $2.48(10)$ \\
\hline & & $501+810$ & & 1309 & 2 & $2.45(10)$ \\
\hline & & $f$ & & 1597 & 7 & $1.89(10)$ \\
\hline & & $2 \cdot 810$ & & 1616 & 4 & $1.85(8)$ \\
\hline & & $186+2 \cdot 810$ & & 1798 & 1 & $1.37(8)$ \\
\hline & & $118+2 \cdot 501+810$ & & 1925 & 1 & $1.15(6)$ \\
\hline & & $810+1597$ & & 2413 & 0.7 & $0.54(3)$ \\
\hline & & $3 \cdot 810$ & & 2428 & 0.3 & $0.53(3)$ \\
\hline
\end{tabular}




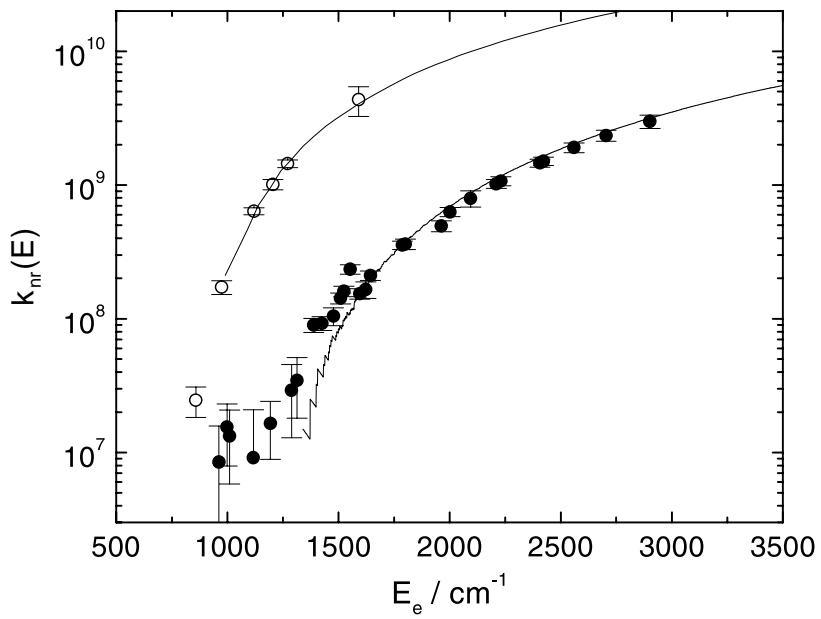

Fig. 9. Excess energy dependence of non-radiative decay rate coefficients $k_{\mathrm{nr}}(E)$ of trans-4 (O) and cis-4 (O). Lines represent the result of RRKM modelling (see text).

intersystem crossing, thus for trans -4 equals $3.73 \pm 0.03 \cdot 10^{8} \mathrm{~s}^{-1}$, in very good agreement with the value reported in room temperature solution [47], while for cis-4 the corresponding value is $k_{0}=(3.31 \pm 0.06) \cdot 10^{8} \mathrm{~s}^{-1}$. The energy dependence of $k_{\mathrm{nr}}\left(E_{\mathrm{e}}\right)$ for both conformers as obtained from (2) is shown in Fig. 9. It can be seen that the threshold for photoisomerization is some $100 \mathrm{~cm}^{-1}$ lower in cis- $\mathbf{4}$ than in trans $\mathbf{4}$ such that the reaction is about an order of magnitude faster for cis $\mathbf{4}$ at the same excess energy.

A quantitative analysis of $k_{\mathrm{nr}}\left(E_{\mathrm{e}}\right)$ in terms of an RRKM fitting procedure as proposed by Troe [20] requires a set of 78 normal mode frequencies for trans $\mathbf{4}$ and cis-4 in the $S_{1}$-state: For this purpose, we calculated equilibrium structures and normal mode frequencies of both conformers in the electronic ground and first excited singlet state using Gaussian98 [72]. Ground state equilibrium bond lengths and angles of both conformers (level: HF 3-21G) are identical to those given in [73]. Upon excitation to the $S_{1^{-}}$ state, they essentially do not change (level: UCIS ST03-G) which is in line with the result that they are also practically identical for trans-4 and cis-4 in both electronic states [73] - as expected, the geometrical structure of $\mathbf{4}$ is fairly rigid. The normal mode frequencies obtained on the basis of these calculations, as listed in Table 3, have to be regarded as preliminary. While the quality of calculations does not yet permit the normal assignment of observed transitions, for the purpose of RRKM modeling the set of frequencies is sufficient as a starting point to extract a barrier height $E_{0}$ for the reaction and to estimate high pressure limits of the thermal rate constants by appropriate integration of $k_{\mathrm{nr}}\left(E_{\mathrm{e}}\right)$ over a Boltzmann distribution $f(E)$ of vibrational energy. 
Table 2. LIF excitation transitions and corresponding fluorescence lifetimes of cis-4.

\begin{tabular}{|c|c|c|c|c|}
\hline $\begin{array}{c}E_{\mathrm{e}} / \mathrm{cm}^{-1} \\
\text { (ns-LIF/jet) }\end{array}$ & $I_{\text {rel. }}$ & $\begin{array}{c}E_{\mathrm{e}} / \mathrm{cm}^{-1} \\
\text { (ps-LIF/jet) }\end{array}$ & $I_{\text {rel. }}$ & $\tau_{\mathrm{fl}} / \mathrm{ns}$ \\
\hline 0 & 100 & 0 & 100 & $3.08(8)$ \\
\hline 62 & 50 & 61 & 8 & $3.06(8)$ \\
\hline 72 & 20 & $72(\mathrm{sh})$ & 2 & \\
\hline 119 & 5 & $106^{*}$ & 4 & \\
\hline 127 & 8 & 125 & 1 & \\
\hline \multirow[t]{20}{*}{132} & 4 & & & \\
\hline & & 293 & 3 & 2.95(8) \\
\hline & & $404 *$ & 2 & \\
\hline & & $518^{*}$ & 1 & \\
\hline & & 560 & 8 & $3.10(10)$ \\
\hline & & 663 & 2 & $2.97(10)$ \\
\hline & & $715^{*}$ & 6 & \\
\hline & & 788 & 12 & $3.03(5)$ \\
\hline & & 851 & 1 & $2.81(5)$ \\
\hline & & 859 & 0.6 & \\
\hline & & 933 & 0.4 & \\
\hline & & 975 & 2 & $1.98(8)$ \\
\hline & & 1116 & 1.2 & $1.03(4)$ \\
\hline & & 1192 & 0.8 & $0.74(5)$ \\
\hline & & 1272 & 0.3 & $0.56(3)$ \\
\hline & & 1348 & 0.3 & \\
\hline & & 1511 & 0.2 & \\
\hline & & 1534 & 0.2 & \\
\hline & & 1576 & 0.3 & \\
\hline & & 1590 & 0.7 & $0.21(5)$ \\
\hline
\end{tabular}

Microcanonical rate constants were calculated according to

$$
k(E)=\frac{W^{\dagger}\left(E-E_{0}\right)}{h \rho(E)}
$$

where $W^{\dagger}$ denotes the number of vibrational states between $E$ and $E_{0}$ in the transition state, $h$ Planck's constant, and $\rho(E)$ the density of vibrational states at energy $E$ in $S_{1}$. Fitting this RRKM model to the experimental data involved two free parameters: the barrier height $E_{0}$ and a frequency scaling factor to adjust a subset of frequencies of the $S_{1}$-state to those in the transition state. In addition, a reactive mode frequency for isomerization in the $S_{1}$-state has to be selected. By inspection of the normal modes obtained from the ab initio calculations, we chose $v_{\text {iso }}=56.7 \mathrm{~cm}^{-1}$ as being strongly correlated with ethylenic 
Table 3. Calculated normal mode frequencies for the $S_{1}$-state used in RRKM modeling microcanonical reaction rates $k_{\mathrm{nr}}(E)$ (see text).

\begin{tabular}{|c|c|c|c|c|c|}
\hline mode & $\begin{array}{l}\tilde{v} / \mathrm{cm}^{-1} \\
\text { trans }-4\end{array}$ & $\begin{array}{c}\tilde{v} / \mathrm{cm}^{-1} \\
\text { cis-4 }\end{array}$ & mode & $\begin{array}{l}\tilde{v} / \mathrm{cm}^{-1} \\
\text { trans }-4\end{array}$ & $\begin{array}{c}\tilde{v} / \mathrm{cm}^{-1} \\
\text { cis-4 }\end{array}$ \\
\hline 1 & 42.2 & 47.0 & 40 & 1119.7 & 1116.4 \\
\hline 2 & 56.7 & 51.5 & 41 & 1127.1 & 1138.0 \\
\hline 3 & 63.3 & 65.5 & 42 & 1137.5 & 1149.1 \\
\hline 4 & 112.6 & 111.4 & 43 & 1149.3 & 1159.1 \\
\hline 5 & 208.0 & 221.6 & 44 & 1181.1 & 1178.4 \\
\hline 6 & 221.9 & 237.1 & 45 & 1191.0 & 1179.1 \\
\hline 7 & 237.3 & 246.2 & 46 & 1198.5 & 1193.9 \\
\hline 8 & 322.8 & 322.6 & 47 & 1204.8 & 1213.9 \\
\hline 9 & 361.3 & 324.2 & 48 & 1217.1 & 1215.7 \\
\hline 10 & 379.5 & 380.8 & 48 & 1242.7 & 1247.1 \\
\hline 11 & 391.3 & 389.7 & 50 & 1282.1 & 1282.2 \\
\hline 12 & 444.6 & 443.8 & 51 & 1318.1 & 1317.5 \\
\hline 13 & 461.7 & 465.0 & 52 & 1322.6 & 1319.6 \\
\hline 14 & 514.0 & 505.0 & 53 & 1377.9 & 1375.6 \\
\hline 15 & 532.4 & 511.4 & 54 & 1386.5 & 1392.6 \\
\hline 16 & 542.5 & 590.8 & 55 & 1392.7 & 1397.2 \\
\hline 17 & 606.2 & 608.6 & 56 & 1521.7 & 1518.6 \\
\hline 18 & 650.6 & 625.1 & 57 & 1535.7 & 1536.7 \\
\hline 19 & 658.5 & 658.5 & 58 & 1541.1 & 1540.1 \\
\hline 20 & 662.7 & 694.0 & 59 & 1545.9 & 1545.6 \\
\hline 21 & 696.5 & 701.7 & 60 & 1596.6 & 1596.1 \\
\hline 22 & 699.5 & 703.4 & 61 & 1617.4 & 1617.8 \\
\hline 23 & 718.7 & 715.6 & 62 & 1622.0 & 1622.0 \\
\hline 24 & 754.3 & 753.0 & 63 & 1644.2 & 1643.4 \\
\hline 25 & 758.3 & 759.9 & 64 & 1646.5 & 1647.0 \\
\hline 26 & 800.9 & 769.3 & 65 & 1661.0 & 1664.2 \\
\hline 27 & 874.8 & 873.2 & 66 & 1968.9 & 1962.8 \\
\hline 28 & 875.2 & 874.7 & 67 & 3250.1 & 3249.4 \\
\hline 29 & 927.7 & 930.0 & 68 & 3250.5 & 3249.8 \\
\hline 30 & 939.6 & 939.4 & 69 & 3355.1 & 3354.3 \\
\hline 31 & 940.4 & 939.5 & 70 & 3355.2 & 3354.4 \\
\hline 32 & 972.1 & 971.6 & 71 & 3367.6 & 3367.8 \\
\hline 33 & 976.3 & 981.7 & 72 & 3368.1 & 3368.5 \\
\hline 34 & 982.0 & 981.9 & 73 & 3380.6 & 3380.9 \\
\hline 35 & 982.0 & 985.2 & 74 & 3380.6 & 3381.2 \\
\hline 36 & 1043.6 & 1016.1 & 75 & 3387.8 & 3387.8 \\
\hline 37 & 1063.4 & 1039.7 & 76 & 3388.4 & 3388.9 \\
\hline 38 & 1071.0 & 1066.0 & 77 & 3390.1 & 3392.0 \\
\hline 39 & 1098.7 & 1113.8 & 78 & 3391.3 & 3395.2 \\
\hline
\end{tabular}

torsion. The curves in Fig. 9 represent the results of modeling both trans $-\mathbf{4}$ and cis-4 excited state photoisomerization. While for cis-4 no frequency scaling was required, for trans $\mathbf{- 4}$ we had to use a scaling factor of 1.03 for the 23 lowest 
frequency modes. The barrier to photoisomerization turned out to be (1340 \pm 50) $\mathrm{cm}^{-1}$ and $(900 \pm 100) \mathrm{cm}^{-1}$ for trans $-\mathbf{4}$ and cis-4, respectively. The former value compares well with the barrier height of $(1400 \pm 100) \mathrm{cm}^{-1}$ determined in non-polar liquid solution [47]. Using the expression

$$
k_{\infty}=\int_{E_{0}}^{\infty} k(E) f(E) \mathrm{d} E,
$$

one then may easily predict the high pressure limit of the thermal rate constant, $k_{\infty}$, from $k(E), E_{0}$ and the normal mode frequencies of the $S_{1}$-states. For $T=$ $300 \mathrm{~K}$ the results are $k_{\infty}=1.8 \cdot 10^{9} \mathrm{~s}^{-1}$ and $k_{\infty}=1.2 \cdot 10^{10} \mathrm{~s}^{-1}$ for trans-4 and cis-4, respectively.

These values can be compared directly with corresponding rate coefficients in room temperature low viscosity liquid or fluid solution. Schneider et al. [47] measured a value of about $8 \cdot 10^{9} \mathrm{~s}^{-1}$ at $T=303 \mathrm{~K}$ for trans -4 in both $n$-hexane and methylcyclohexane solution. However, they did not take cis-4 fluorescence into account in their lifetime analysis. Therefore, we measured the fluorescence decay of a solution of the conformer mixture and of pure trans- 4 in $n$-hexane at room temperature using our single photon counting set-up and analyzed it using Brochon's fit program based on a maximum entropy method lifetime analysis [74]. We arrived at fluorescence lifetimes of $(200 \pm 10)$ ps and $(75 \pm 10)$ ps for trans $\mathbf{- 4}$ and cis $\mathbf{- 4}$, respectively. Assuming that the sum of contributions from radiative decay, internal conversion, and intersystem crossing does not change between jet-cooled molecule conditions and liquid solution, these lifetimes correspond to photoisomerization rate constants of $4.6 \cdot 10^{9} \mathrm{~s}^{-1}$ for trans $\mathbf{- 4}$ and $1.3 \cdot 10^{10} \mathrm{~s}^{-1}$ for cis-4. These rate coefficients should be close to the hypothetical high pressure limit, because the viscosity of liquid $n$-hexane at $300 \mathrm{~K}$ is fairly low and will not slow down the reaction rate by more than a factor of two from its zero friction value [48]. Therefore, bearing in mind the uncertainties of modeling, one may conclude that while for $\mathrm{cis}-\mathbf{4}$ the model prediction is in agreement with the experimental result, there is a notable discrepancy for trans $\mathbf{- 4}$ which may be either due to deficiencies of the model or indicate solvation and/or dynamic effects similar to those still under controversial discussion for trans-1. One should note, however, that for trans-1 the difference between the RRKM prediction of the rate coefficient and its measured value in low viscosity fluid solution is about a factor of 50 [75], while here it is at most a factor of 4 to 5. So one should really try to improve the harmonic model first - with the immediate aim to quantitatively assign the LIF excitation spectrum, before one embarks on a detailed discussion of conceivable physical effects.

In another approach to experimentally determine the high pressure limit of the photoisomerization rate coefficient of trans-4, we presently measure its fluorescence decay in thermal vapor and with added buffer gas, again in the single 


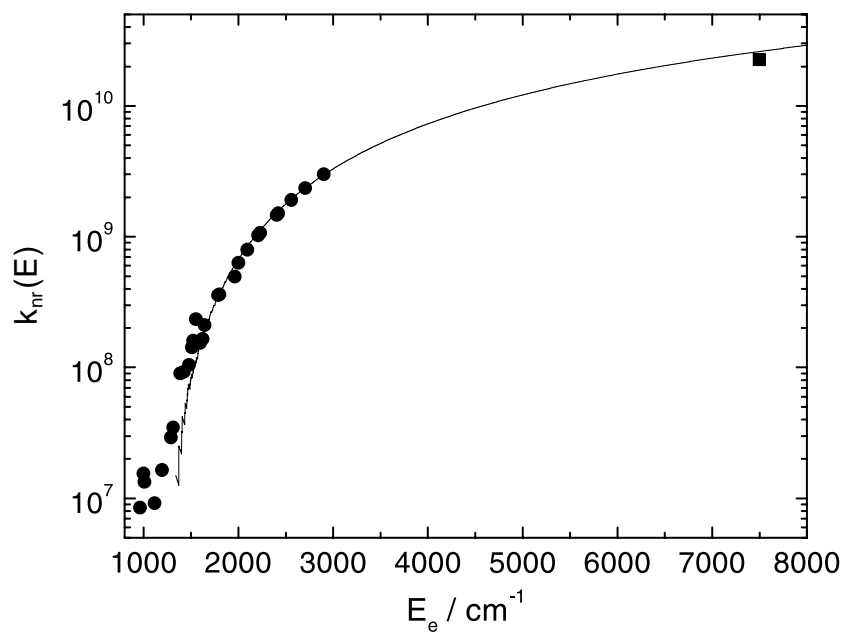

Fig. 10. Excess energy dependence of non-radiative decay rate coefficients $k_{\mathrm{nr}}(E)$ of trans $\mathbf{4}(-)$ and rate coefficient measured in thermal trans $\mathbf{4}$ vapor at $300 \mathrm{~K}(\boldsymbol{\square})$. Line as in Fig. 9.

photon counting set-up described above using a suitable sample cell $[49,76]$. First preliminary experiments indicate that in thermal trans-4 vapor under collision free conditions the decay consists of two components: a dominant fast one contributing about 95 percent to the signal amplitude that may be characterized by a lifetime of $47 \mathrm{ps}$, and a slow component whose time constant is about 10 times as large. We are convinced that the fast component reflects the decay of the initially excited population in the $S_{1}$-state which is just the thermal population of the ground state lifted by the $266 \mathrm{~nm}$ excitation photon to a corresponding excess energy in $S_{1}$. The sum of the mean thermal energy at $300 \mathrm{~K}$ (about $2000 \mathrm{~cm}^{-1}$ ) and the photon energy $\left(37600 \mathrm{~cm}^{-1}\right)$ minus the energy of the $0-0$-transition of trans -4 is about $7500 \mathrm{~cm}^{-1}$, i.e. most of the molecules will react with a rate corresponding to this internal energy resulting in a practically mono-exponential fast decay component. The measured rate constant of $2.1 \cdot 10^{10} \mathrm{~s}^{-1}$ is very close to the RRKM model prediction of $2.5 \cdot 10^{10} \mathrm{~s}^{-1}$ as shown in Fig. 10.

The origin of the slow component has to be clarified in further experiments. It could involve fast forward and slow reverse intersystem crossing between $S_{1}$ and $T_{1}$ at high excess energy or, more likely, reflect consequences of simultaneous excitation of a higher singlet state $\left(\mathrm{A}^{\prime}\right)$ which is accessible at this excitation wavelength [73]. We have also started to study the effect of added bath gas. Once the pressure of the bath gas is sufficiently high to rapidly cool the initially vibrationally hot molecule and subsequently sustain an equilibrium distribution in the $S_{1}$-state, the fast component should disappear and one should measure the high pressure limit of the fluorescence decay rate con- 
stant. At pressures of 8 bar $n$-propane we have not yet reached this situation, but the fast component is reduced to about two thirds of the signal amplitude while a slower component with a life time of about 300 ps has become more prominent. This is a very strong indication that the high pressure limit of the photoisomerization rate constant will be very close to the value we measured in liquid $n$-hexane. The discrepancy between the predicted $k_{\infty}$ and the corresponding experimentally determined rate coefficient, therefore, is unlikely to be more than a factor of three and almost within the error limits expected from such simple models.

\section{Conclusion}

We presented first measurements of fluorescence excitation spectra of the rigid stilbene analogue $\mathbf{4}$ in a supersonic jet expansion showing that, in contrast to the parent compound, both the trans- and the cis-conformer fluoresce under these conditions. The excess energy dependence of fluorescence lifetimes indicates the onset of an efficient non-radiative decay channel above energy thresholds of $1340 \mathrm{~cm}^{-1}$ and $990 \mathrm{~cm}^{-1}$ for the trans- and cis-form, respectively, which is assigned to photoisomerization in the singlet state. From an RRKM analysis of the microcanonical rate coefficients an estimate of the high pressure limit of the thermal photoisomerization rate coefficient is obtained and compared with photoisomerization rate coefficients measured in low viscosity solution and in thermal vapor. There are strong indications that for this compound there are no dynamic or static solvent induced effects that lead to an anomalous acceleration of the reaction in solution. If this conclusion will be, as we expect, also be verified in studies underway in fluid solution, trans-1 still is the only compound of this family for which peculiar discrepancies between isolated and "solvated" molecule reaction dynamics have been found.

\section{Acknowledgement}

The authors thank Professor Siegfried Schneider and Dr. Martin Votsmeier for many helpful discussions in early stages of this project, and the Deutsche Forschungsgemeinschaft for financial support in the framework of the Sonderforschungsbereich 357 "Molekulare Mechanismen unimolekularer Prozesse".

\section{References}

1. J. Saltiel, J. T. D’Agostino, E. D. Megarity, L. Metts, K. R. Neuberger, M. Wrighton and O. C. Zafiriou, Org. Photochem. 3 (1973) 1.

2. J. Saltiel and J. L. Charlton, in: Rearrangement in Ground and Excited States, P. deMayo, ed., p. 25, Academic Press, New York 1980.

3. R. M. Hochstrasser, Int. J. Pure \& Appl. Chem. 52 (1980) 2683.

4. G. R. Fleming, D. H. Waldeck, K. M. Keery, and S. P. Velsko, in: Applications of Picosecond Spectroscopy to Chemistry, 1984. 
5. J. Schroeder and J. Troe, Ann. Rev. Phys. Chem. 38 (1987) 163.

6. J. Saltiel and Y.-P. Sun, in: Photochromism, Molecules, and Systems, eds. H. Dürr and H. Bouas-Laurent, p. 64, Elsevier, Amsterdam 1990.

7. D. H. Waldeck, Chem. Rev. 91 (1991) 415.

8. D. H. Waldeck, J. Molec. Liq. 57 (1993) 127.

9. J. Schroeder and J. Troe, in: Activated Barrier Crossing, eds. G. R. Fleming and P. Hänggi, p. 206, World Scientific, Singapore 1993.

10. H. Görner and H. J. Kuhn, Adv. Photochem. 19 (1995) 1.

11. G. A. Voth and R. M. Hochstrasser, J. Phys. Chem. 100 (1996) 13034.

12. J. Schroeder, Ber. Bunsenges. Phys. Chem. 101 (1997) 643.

13. G. Rothenberger, D. K. Negus and R. M. Hochstrasser, J. Chem. Phys. 79 (1983) 5360 .

14. V. Sundström and T. Gillbro, Chem. Phys. Lett. 109 (1984) 538.

15. J. Schroeder, J. Troe and P. Vöhringer, Chem. Phys. Lett. 203 (1993) 255.

16. G. Maneke, J. Schroeder, J. Troe and F. Voss, Ber. Bunsenges. Phys. Chem. 89 (1985) 896.

17. J. Schroeder and J. Troe, Chem. Phys. Lett. 116 (1985) 453.

18. M. Lee, G. R. Holtom and R. M. Hochstrasser, Chem. Phys. Lett. 118 (1985) 359.

19. L. R. Khundkar, R. A. Marcus and A. H. Zewail, J. Phys. Chem. 87 (1983) 2473.

20. J. Troe, Chem. Phys. Lett. 114 (1985) 241.

21. J. Schroeder and J. Troe, J. Phys. Chem. 90 (1986) 4215.

22. J. A. Syage, W. M. Lambert, P. M. Felker, A. H. Zewail and R. M. Hochstrasser, Chem. Phys. Lett. 88 (1982) 266.

23. A. Amirav and J. Jortner, Chem. Phys. Lett. 95 (1983) 295.

24. J. A. Syage, P. M. Felker and A. H. Zewail, J. Chem. Phys. 81 (1984) 4706.

25. J. A. Syage, P. M. Felker and A. H. Zewail, J. Chem. Phys. 81 (1984) 4685.

26. T. J. Majors, U. Even and J. Jortner, J. Chem. Phys. 81 (1984) 2330.

27. S. P. Velsko and G. R. Fleming, J. Chem. Phys. 76 (1982) 3553.

28. C. Gehrke, R. Mohrschladt, J. Schroeder, J. Troe and P. Vöhringer, Chem. Phys. 152 (1991) 45.

29. R. Mohrschladt, J. Schroeder, D. Schwarzer, J. Troe and P. Vöhringer, J. Chem. Phys. 101 (1994) 7566.

30. H. A. Kramers, Physica 7 (1940) 284.

31. J. Troe, in: High pressure chemistry, eds. H. Kelm, p. 489, D. Reidel, Amsterdam 1978.

32. J. F. Shepanski, B. W. Keelan and A. H. Zewail, Chem. Phys. Lett. 103 (1983) 9.

33. J. Troe, A. Amirav and J. Jortner, Chem. Phys. Lett. 115 (1985) 245.

34. A. Amirav, M. Sonnenschein and J. Jortner, Chem. Phys. 102 (1986) 305.

35. C. Gehrke, J. Schroeder, D. Schwarzer, J. Troe and F. Voss, J. Chem. Phys. 92 (1990) 4805.

36. A. Gierer and K. Wirtz, Z. Naturf. 8a (1953) 532.

37. J. L. Dote, D. Kivelson and R. N. Schwartz, J. Phys. Chem. 85 (1981) 2169.

38. M. Lee, A. J. Bain, P. J. McCarthy, C. H. Han, J. N. Haseltine, A. B. Smith III and R. M. Hochstrasser, J. Chem. Phys. 85 (1986) 4341.

39. S. H. Courtney, S. K. Kim, S. Canonica and G. R. Fleming, J. Chem. Soc. Faraday Trans. 282 (1986) 2065.

40. Y.-P. Sun and J. Saltiel, J. Phys. Chem. 93 (1989) 8310.

41. S. K. Kim and G. R. Fleming, J. Phys. Chem. 92 (1988) 2168.

42. L. Nikowa, D. Schwarzer, J. Troe and J. Schroeder, J. Chem. Phys. 97 (1992) 4827.

43. J. Schroeder, J. Phys. Cond. Matter 8 (1996) 9379.

44. R. Mohrschladt, J. Schroeder, J. Troe, P. Vöhringer and M. Votsmeier, 60 Ultrafast Phenomena IX (1994) 499. 
45. H. Petek, Y. Fujiwara, D. Kim and K. Yoshihara, J. Am. Chem. Soc. 110 (1988) 6269.

46. R. Nörenberg, Diploma-Thesis, Universität Göttingen, 1993.

47. S. Schneider, B. Brem, W. Jäger, H. Rehaber, D. Lenoir and R. Frank, Chem. Phys. Lett. 308 (1999) 211.

48. J. Schroeder, J. Troe and P. Vöhringer, Z. Phys. Chem. 188 (1995) 287.

49. A. Meyer, J. Schroeder and J. Troe, J. Phys. Chem. A 103 (1999) 10528.

50. R. S. Larson and M. D. Kostin, J. Chem. Phys. 72 (1980) 1392.

51. H. A. Weidenmüller and J.-S. Zhang, J. Stat. Phys. 34 (1984) 191.

52. B. Carmeli and A. Nitzan, Phys. Rev. A 29 (1984) 1481.

53. M. Borkovec and B. J. Berne, J. Chem. Phys. 86 (1987) 2444.

54. J. Lee, S.-B. Zhu and G. W. Robinson, J. Phys. Chem. 91 (1987) 4273.

55. B. J. Matkowsky, A. Nitzan and Z. Schuss, J. Chem. Phys. 88 (1988) 4765.

56. A. M. Berezhkovskii, L. M. Berezhkovskii and V. Yu. Zitserman, Chem. Phys. 130 (1989) 55.

57. M. M. Klosek-Dygas, B. M. Hoffman, B. J. Matkowsky, A. Nitzan, M. A. Ratner and Z. Schuss, J. Chem. Phys. 90 (1989) 1141.

58. A. M. Berezhkovskii and V. Yu. Zitserman, Chem. Phys. Lett. 212 (1993) 413.

59. A. M. Berezhkovskii, A. M. Frishman and E. Pollak, J. Chem. Phys. 101 (1994) 4778.

60. N. Agmon and R. Kosloff, J. Phys. Chem. 91 (1987) 1988.

61. J. Troe and K.-M. Weitzel, J. Chem. Phys. 88 (1988) 7030.

62. C. Lienau, J. Schroeder, J. Troe and K. Wack, Ber. Bunsenges. Phys. Chem. 101 (1997) 614.

63. R. Daum, T. Hansson, R. Nörenberg, D. Schwarzer and J. Schroeder, Chem. Phys. Lett. 246 (1995) 607.

64. J. W. Barton and M. K. Shepherd, J. Chem. Soc. Perkin Trans. I 1987, 1561.

65. W. R. Roth, V. Staemmler, M. Neumann and C. Schmuck, Liebigs Ann. Org. Bioorg. Chem. 6 (1995) 1061.

66. A. Frimer, J. Weiss and Z. Rosental, J. Org. Chem. 59 (1994) 2516.

67. J. McMurry, Chem. Rev. 89 (1989) 1513.

68. D. Lenoir, Synthesis 1989, 883.

69. H. Petek, K. Yoshihara, Y. Fujiwara and J. G. Frey, J. Opt. Soc. Am. B7 (1990) 1540.

70. G. Hohlneicher, I. Kautz, B. Tillmanns, D. Lenoir and R. Frank, J. Mol. Struct. 348 (1995) 191.

71. J. A. Syage, F. Al Adel and A. H. Zewail, Chem. Phys. Lett. 103 (1983) 15.

72. Gaussian98 by M. J. Frisch, G. W. Trucks, H. B. Schlegel, G. E. Scuseria, M. A. Robb， J. R. Cheeseman, V. G. Zakrzewski， J. A. Montgomery, R. E. Stratmann, J. C. Burant, S. Dapprich, J. M. Millam, A. D. Daniels, K. N. Kudin, M. C. Strain, O. Farkas, J. Tomasi, V. Barone, M. Cossi, R. Cammi, B. Mennuci, C. Pomelli, C. Adamo, S. Clifford, J. Ochterski, G. A. Petersson, P. Y. Ayala, Q. Cui, K. Morokuma, D. K. Malick, A. D. Rabuck, K. Raghavachari, J. B. Foresman, J. Cioslowski, J. V. Ortiz, B. B. Stefanov, G. Liu, A. Liashenko, P. Piskorz, I. Komaromi, R. Gomperts, R. L. Martin, D. J. Fox, T. Keith, L. A. Al-Laham, C. Y. Peng, A. Nanayakkara, C. Gonzales, M. Challacombe, P. M. W. Gill, G. B. Johnson, W. Chen, M. W. Wong, J. L. Andres, M. Head-Gordon, E. S. Replogle and J. A. Pople; Gaussian Inc., 1998.

73. G. Hohlneicher, R. Wrzal, D. Lenoir and R. Frank, J. Phys. Chem. A 103 (1999) 8969.

74. FLAME5 by J.-C. Brochon; MaxEnt Solutions Ltd., 1998.

75. J. Schroeder, Ber. Bunsenges. Phys. Chem. 95 (1991) 233.

76. A. Meyer, J. Schroeder, J. Troe and M. Votsmeier, J. Photochem. Photobiol. A: Chemistry 105 (1997) 345. 